\title{
BMJ Open Pilot randomised controlled trial of a patient navigation intervention to enhance engagement in the PrEP continuum among young Latino MSM: a protocol paper
}

Aaron J Blashill, ${ }^{1,2}$ Janna R Gordon, ${ }^{2}$ Sarah A Rojas (D) , ${ }^{3}$ Christian B Ramers, ${ }^{3}$ Chii-Dean Lin, ${ }^{1}$ Claudia M Carrizosa, ${ }^{4}$ Kelsey A Nogg, ${ }^{4}$ Kalina M Lamb, ${ }^{1}$ Nicholas C Lucido (D) , 'Isaiah J Jones, ${ }^{1}$ David Rivera, ${ }^{1}$ Rosa A Cobian Aguilar, ${ }^{1}$ John P Brady, ${ }^{2}$ Martin Fuentes, ${ }^{3}$ Kristen J Wells ${ }^{1,2}$

To cite: Blashill AJ, Gordon JR, Rojas SA, et al. Pilot randomised controlled trial of a patient navigation intervention to enhance engagement in the PrEP continuum among young Latino MSM: a protocol paper. BMJ Open 2021;11:e040955. doi:10.1136/ bmjopen-2020-040955

- Prepublication history and additional supplemental material for this paper are available online. To view these files, please visit the journal online (http://dx.doi.org/10.1136/ bmjopen-2020-040955).

Received 27 May 2020 Revised 09 January 2021 Accepted 16 March 2021

Check for updates

(C) Author(s) (or their employer(s)) 2021. Re-use permitted under CC BY-NC. No commercial re-use. See rights and permissions. Published by BMJ.

For numbered affiliations see end of article.

Correspondence to

Dr Kristen J Wells;

kwells@mail.sdsu.edu

\section{ABSTRACT}

Introduction Men who have sex with men (MSM) are one of the most at-risk group for contracting HIV in the USA. However, the HIV epidemic impacts some groups of MSM disproportionately. Latino MSM comprise $25.1 \%$ of new HIV infections among MSM between the ages of 13 and 29 years. The daily medication tenofovir/emtricitabine was approved by the Food and Drug Administration for preexposure prophylaxis (PrEP) in 2012 and has demonstrated strong efficacy in reducing HIV acquisition.

Methods and analysis Through extensive formative research, this study uses a pilot randomised controlled trial design and will examine the feasibility and acceptability of a patient navigation intervention designed to address multiple barriers to improve engagement in the PrEP continuum among 60 Latino MSM between the ages of 18 and 29 years. The patient navigation intervention will be compared with usual care plus written information to evaluate the feasibility and acceptability of the intervention and study methods and the intervention's potential in improving PrEP continuum behaviours. The results will be reviewed for preparation for a future full-scale efficacy trial.

Ethics and dissemination This study was approved by the institutional review board at San Diego State University and is registered at ClinicalTrials.gov. The intervention development process, plan and the results of this study will be shared through peer-reviewed journal publications, conference presentations and healthcare system and community presentations.

Registration details Registered under the National Institutes of Health's ClinicalTrials.gov (NCT04048382) on 7 August 2019 and approved by the San Diego State University (HS2017-0187) institutional review board. This study began on 5 August 2019 and is estimated to continue through 31 March 2021. The clinical trial is in the pre-results stage.

\section{INTRODUCTION}

Reducing the number of new HIV infections is one of the goals of the National HIV/ AIDS Strategy and an objective of Healthy

\section{Strengths and limitations of this study}

- There are no other known patient navigation trials targeting the pre-exposure prophylaxis (PrEP) cascade.

- The trial will target each step of the PrEP cascade.

- The trial will use peers as interventionists, which may increase scalability.

- The sample size of the pilot trial is underpowered to fully test the efficacy of the intervention.

People 2020. ${ }^{12}$ Men who have sex with men (MSM) are one of the most at-risk group for contracting HIV in the USA, comprising $67 \%$ of new diagnoses in $2016 .{ }^{3}$ It is estimated that $24.7 \%$ of sexually active MSM are at substantial risk for acquiring HIV. ${ }^{4}$ The HIV epidemic impacts some groups of MSM disproportionately, with Latino MSM comprising $25.1 \%$ of new HIV infections among MSM between 13 and 29 years of age. ${ }^{5}$ Recent modelling studies suggest that one out of four Latino MSM will be diagnosed with HIV within their lives, underscoring the vulnerability of Latino MSM to HIV infection. ${ }^{3}$

Pre-exposure prophylaxis (PrEP), a biomedical tool to reduce risk of HIV infection, has shown strong efficacy. When taken consistently, PrEP can reduce new incidence of HIV by up to $99 \% .{ }^{6}$ Despite the promise of PrEP to transform HIV prevention, there remain significant barriers to PrEP use among those who would benefit most, including low awareness, low knowledge, medical mistrust, perceived high cost, lack of access, language barriers, low health literacy, cultural conceptions of masculinity and stigma. ${ }^{7-12}$ In a 2016 
study conducted among 600 MSM between the ages of 18 and 29 years in Southern California, Latino MSM reported the lowest levels of PrEP use at 6.6\%, compared with $9.8 \%$ and $13.9 \%$ for black and white participants, respectively. ${ }^{7}$ Although Latino MSM reported the lowest use of PrEP, they also reported the greatest willingness to use PrEP compared with white and black MSM. This discrepancy in willingness versus PrEP utilisation among Latino MSM emphasises the need to intervene to address barriers among this population.

There are a number of different steps that individuals must take to initiate, adhere to and persist in taking PrEP. Individuals must be aware of their HIV risk and eligibility for PrEP, be interested in taking PrEP, be linked to PrEP-related care, initiate PrEP-related care, be retained in PrEP-related care and maintain adherence to PrEP, with each step in this continuum of care associated with unique barriers. ${ }^{13}$ One approach that has been used to address multiple barriers to adhering to recommended healthcare is patient navigation (PN). Patient navigators work with the individual patient, the patient's significant others, the healthcare system and community services to reduce delays and improve adherence in healthcare. PN has facilitated access to healthcare among other Latino populations, ${ }^{14-16}$ and peer PN may be an ideal intervention to aid in PrEP uptake among Latino MSM by assisting individuals in overcoming barriers to PrEP-related care. Thus, our research team has developed a peer PN intervention. To our knowledge, no known PN programmes for PrEP have been developed or pilot tested specifically for Latino MSM. This paper describes the design of a study to test a recently developed PN intervention to increase engagement in the PrEP continuum among Latino MSM.

\section{METHODS AND ANALYSIS}

\section{Study design}

Using a randomised controlled trial design, a pilot test of the PN intervention will be conducted in collaboration with the Family Health Centers of San Diego (FHCSD), a federally qualified health centre. ${ }^{17}$ Bilingual research assistants will recruit 60 young adult Latino MSM at-risk for HIV in San Diego County, California; this sample size is well within the bounds of recommendations for pilot trials. ${ }^{18}$ After a research assistant obtains informed consent, participants will complete baseline surveys and the research assistant will individually randomise each participant to one of two conditions: usual care plus written information (UC) or PN. At 3-month and 6-month follow-up, participants will be asked to complete surveys. Individuals randomised to $\mathrm{PN}$ will also be asked to participate in a key informant interview at 6 months. Participants' electronic health records (EHRs) will be reviewed at 6-month follow-up to assess their engagement in the PrEP continuum. The study methods and PN intervention will be reviewed for feasibility, acceptability, and the preliminary impact of the PN intervention in preparation for a future full-scale efficacy trial. In addition, the pilot test will evaluate preliminary impact on seven PrEP continuum-related primary outcomes (i.e., scheduled and attended PrEP consultation; PrEP prescription received; PrEP prescription filled; PrEP initiated; selfreported PrEP adherence; and PrEP follow-up medical appointment attended). Analyses will be performed under the intent-to-treat principle. Participants will be compensated with a $\$ 50$ gift card to an online retailer at each of the three assessment time points (baseline, 3-month follow-up and 6-month follow-up) (see online supplemental appendices 1-3 for informed consents and WHO Trial Registration Data Set).

\section{Patient and public involvement}

As suggested by intervention mapping, a participatory planning group (PPG) of programme stakeholders guided the design of the PN intervention. The PPG includes a consultant and HIV expert, potential programme implementers, young Latino MSM and community members who interact with or deliver services to people who may benefit from the intervention. The PPG was instrumental in helping us develop the intervention materials and recruitment approaches. This group will continue to meet on an as-needed basis (with compensation for their time) and will assist with dissemination of research findings both in the community and in peer-reviewed publications and presentations.

\section{Setting}

The pilot RCT will take place in San Diego County, California. In 2017, San Diego had an estimated population of $3,337,685$ people ${ }^{19} 33.9 \%$ of whom identified as Hispanic and 50.3\% identified as male. Between 2012 and 2014, it was estimated that $3.9 \%$ of San Diego County's population identified as a sexual minority. ${ }^{20}$ Our study is being conducted in partnership with FHCSD, which is San Diego's largest federally qualified health centre, providing care to more than 140,834 patients in 2018. FHCSD delivers comprehensive healthcare in 42 sites throughout San Diego County. Each site provides a wide range of healthcare services to sexual and gender minority patients. Although we are partnering with FHCSD on this study, participants are recruited throughout San Diego County and obtain healthcare in various health systems, centres and practices in the community.

\section{Inclusion/exclusion criteria}

The following criteria are required for study inclusion: (1) age 18-29 years; (2) identifies as male; (3) identifies as gay/bisexual or reports having sex with men in past 12 months; (4) identifies as Latino/Hispanic; (5) selfreports being HIV uninfected; (6) resides in San Diego County, California; (7) speaks English or Spanish; (8) is willing and able to provide informed consent; and (9) reports at least one HIV risk factor as informed by Centers for Disease Control and Prevention (CDC) guidelines. ${ }^{21}$ MSM are at elevated risk for HIV if they report one of the following: (1) an HIV-positive sexual partner; 
(2) diagnosis of a bacterial sexually transmitted infection (STI) within the past 12 months; (3) engaging in condomless anal sex with a non-monogamous partner in the past 12 months; (4) engaging in commercial sex work in the past 12 months; (5) injection of illicit drugs and sharing of injection equipment in the past 12 months; or (6) engaging in drug treatment for injection drug use in the past 12 months. Individuals will be excluded if they self-report being HIV positive. The intervention is designed to meet the needs of MSM who self-report their gender identity as male, but other gender identities are allowed in the study. If a potential participant indicates that their gender identity is not 'male' (e.g., transgender, non-binary and gender fluid), then the participant will be informed that the study was designed for cis-male MSM and will then be invited to determine whether they feel that they will benefit from the study based on their gender identity. Participants who previously were prescribed or are currently taking PrEP will be included in the study. Participants are not required to seek care at FHCSD to be included in the study.

\section{Recruitment}

Non-probability (convenience) sampling will be used to recruit participants within the greater San Diego area via a variety of methods, including through FHCSD outreach and HIV/STI testing programmes, a Spanish language Latino MSM support group, gay-friendly events, such as Pride, and flyering local lesbian, gay, bisexual, and transgender (LGBT) community centres, gay-identified/ friendly coffee shops, gyms and bars. We will also use online recruitment methods, including targeted ads through Facebook and Instagram and the use of geolocation social networking mobile applications tailored to sexual minority men (eg, Grindr, Scruff and Jack'd). Potential participants recruited using flyering or targeted ads will be requested to contact study personnel via email or telephone. A bilingual member of the study team will contact each potential participant via telephone to explain the study and screen for inclusion. The research assistants will be native Spanish speakers who are bilingual in English and Spanish; they will have some university education and research experience. If a potential participant screens positively for the study, an in-person baseline visit will be scheduled, in which the inclusion/ exclusion criteria will be assessed in further detail. Reasons for non-participation of potential participants will be documented.

\section{Randomisation}

The research coordinator will use the Randomizer for Clinical Trial computer application to randomise each participant to either UC or PN via an $\mathrm{iPad}^{22}$ immediately following the baseline survey data collection. Randomizer for Clinical Trial will implement a blocked randomisation sequence (in blocks of four participants) to balance randomisation across the arms of the study. The randomisation sequence will be concealed from all members of the study team prior to randomisation.

\section{Usual care intervention}

Immediately following randomisation, the research team member will provide participants randomised to UC the CDC's two-page PrEP Information Sheet in the participant's preferred language (either English or Spanish). This two-page booklet includes the following information: (1) overview of PrEP; (2) eligibility for PrEP; (3) efficacy of PrEP; (4) safety of PrEP; and (5) obtaining, initiating and adhering to PrEP. Participants will also be provided with both verbal and written information regarding available sexual health and HIV prevention services, including PrEP, at FHCSD. Comprehensive HIV-prevention healthcare, including PrEP, is available to study participants at FHCSD at no or minimal cost.

\section{PN intervention}

Based on extensive formative research and using intervention mapping, ${ }^{23}$ the study team developed, pretested and produced the PN intervention materials. The produced PN intervention includes an introductory module, five educational modules (HIV prevention, PrEP introduction, PrEP efficacy, PrEP side effects and PrEP adherence) and a module focused on decision support. Patient education is facilitated using infographics, palm cards and a decision support tool. The educational modules are designed to be delivered as needed in addition to personalised strategies to improve access and decrease barriers to PrEP initiation and adherence. The intervention also consists of barrier reduction strategies to assist individuals with implementing HIV prevention, including the use of PrEP. Two part-time Spanish-English bilingual peer lay navigators hired by FHCSD will provide the PN intervention during the study. Navigators were hired based on their familiarity, as well as cultural and linguistic competence, with the intended audience, young Latino MSM. The study team will train the patient navigators using training manuals developed in formative research and via the Patient Navigation Research Program training approach. $^{24}$

Following patient randomisation, the research coordinator will notify the patient navigators regarding the patient's assignment to PN. A patient navigator will contact the participant to schedule the first in-person meeting at a time and location of the participant's convenience. The navigators will provide services to patients using PN intervention manuals and materials the team has developed (see figure 1 for examples of PN services provided at each level of PrEP continuum). Services will generally focus on: (1) overcoming community, health system, interpersonal and individual barriers to accessing PrEP-related healthcare; (2) increasing each patient's knowledge, attitudes and self-efficacy for initiating and adhering to PrEP; (3) improving communication between the patient and healthcare team through appointment scheduling and reminders; and (4) sexual risk reduction 


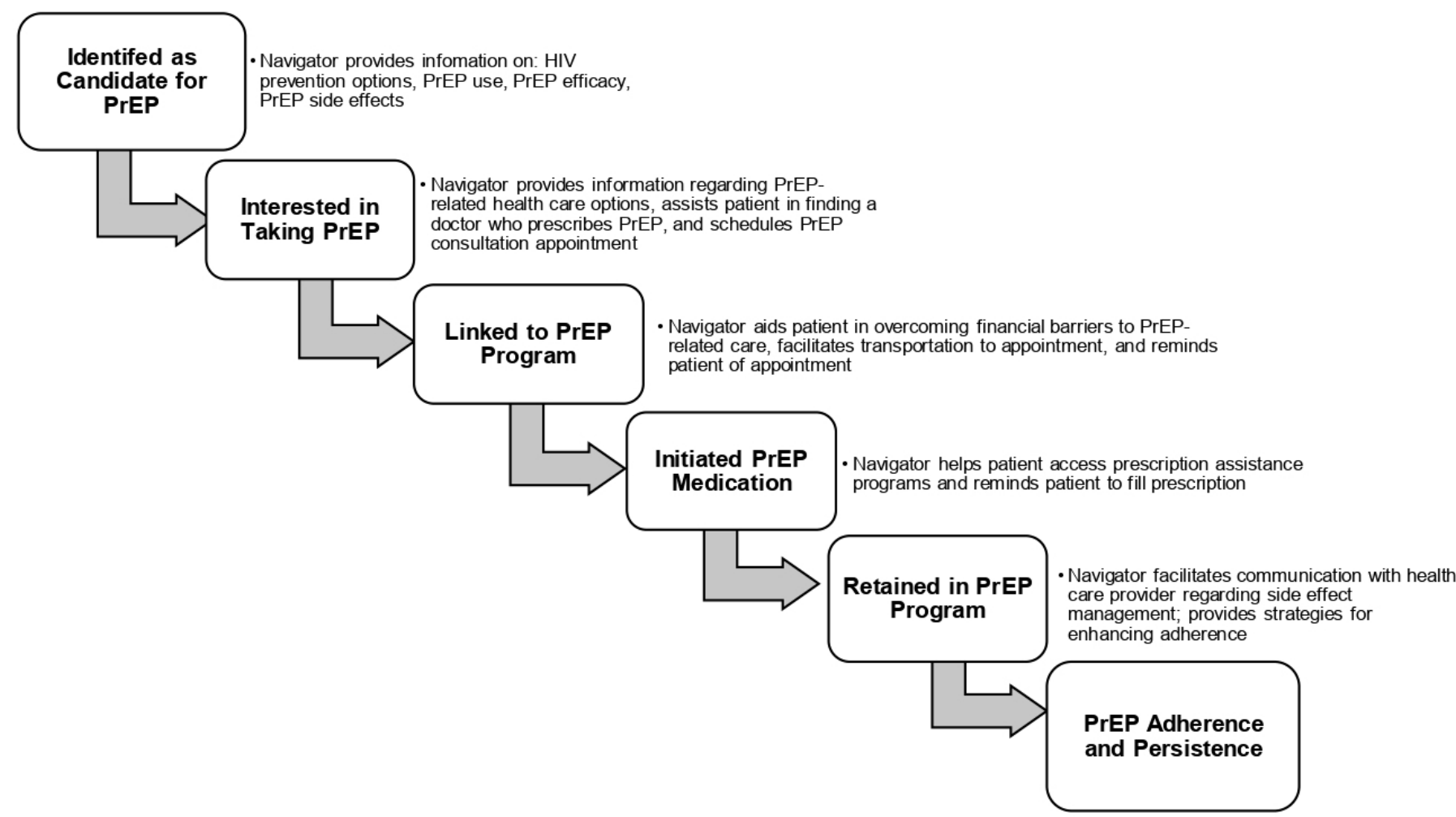

Figure 1 Potential patient navigation intervention processes at each level of the PrEP continuum (PrEP continuum varies between the top box, 'identified as candidate for PrEP', and the bottom box, 'PrEP adherence and persistence'). PrEP, preexposure prophylaxis.

counselling. PNs do not meet participants at their homes but at any safe location to conduct the intervention (e.g., private rooms in a library, clinic or community outreach centre). Meeting locations are coordinated between the participants and the PNs. PNs communicate with participants via text, phone call and videoconferencing based on the participant's preference.

The FHCSD HIV services programme manager will provide day-to-day supervision of the patient navigators and will meet with each navigator weekly and document successes and challenges to PN intervention implementation through a weekly written report. The study staff also provide weekly supervision and will rate patient navigators' delivery of the intervention, via audio recordings, using an intervention fidelity monitoring form.

\section{DATA COLLECTION}

\section{Survey and interview data collection}

Following informed consent, the research coordinator will administer self-report baseline surveys using Qualtrics in-person in the participant's preferred language of Spanish or English, assess time required to complete surveys and note any participant difficulty in answering survey items. Participants will be asked to complete 3-month and 6-month follow-up surveys using a mixed mode approach, consisting of an initial Qualtrics (online) survey followed by an emailed survey if the Qualtrics survey is not completed. At baseline, 3-month follow-up and 6 month follow-up, participants will be asked to complete measures of PrEP knowledge, PrEP awareness, PrEP attitudes, PrEP barriers, PrEP efficacy beliefs, PrEP adherence, PrEP initiation, sexual behaviours, beliefs about medications, medication self-efficacy, mental health, substance use and social support (see table 1 for information regarding study surveys, including evidence of their validity and reliability). Self-report demographic information will be obtained at baseline. Self-report surveys assessing client satisfaction with PrEP-related healthcare services ${ }^{25}$ will be administered at 3-month and 6-month follow-up. Those randomised to PN will complete a selfreport survey assessing satisfaction with the interpersonal relationship with the patient navigator ${ }^{26}$ at 3 months and 6 months. In addition, all participants randomised to PN will be contacted by telephone at 6 months to complete a key informant telephone interview to assess acceptability of the intervention; these key informant interviews will be conducted by members of the assessment team and not by PNs.

Measures were chosen following a literature review of existing validated instruments and psychometrically validated instruments were chosen when possible. However, as PrEP was only recently recommended for the prevention of HIV, there are few validated surveys measuring constructs related to PrEP. For these constructs, the team created new measures, adapting from existing scales when possible. Surveys not available in Spanish were translated using a dual panel approach. ${ }^{27-29}$ 
Table 1 Description and timing of surveys and health record data collection

\begin{tabular}{|c|c|c|c|c|c|c|}
\hline Construct & Description of measure & Source & BL & $3 m$ & $6 m$ & 6m EHR \\
\hline PrEP knowledge & $\begin{array}{l}\text { 16-item self-report measure of knowledge } \\
\text { related to PrEP, including use and } \\
\text { effectiveness. Data are collected using a Likert } \\
\text { scale. }\end{array}$ & Team developed & $x$ & $x$ & $x$ & \\
\hline PrEP awareness & $\begin{array}{l}\text { One survey question assessing awareness of } \\
\text { PrEP. Categorical data are collected. }\end{array}$ & Team developed & $x$ & $x$ & $x$ & \\
\hline PrEP attitudes & $\begin{array}{l}\text { 10-item PrEP Stigma and Positive Attitudes } \\
\text { Measure assessing personal opinions about } \\
\text { PrEP and people who use PrEP; } 16 \text { additional } \\
\text { items assessing general attitudes and beliefs } \\
\text { about PrEP use, provider comfort and } \\
\text { medical mistrust related to PrEP use, side } \\
\text { effect concerns about PrEP use, disclosure } \\
\text { concerns and social norms related to PrEP } \\
\text { use and sexual risk concerns about PrEP use. } \\
\text { Data are collected using a Likert scale. }\end{array}$ & $\begin{array}{l}\text { Mustanski et al, } 2018^{38} \\
\text { Pulsipher et al, },^{7} 2016\end{array}$ & $x$ & $x$ & $x$ & \\
\hline
\end{tabular}

\begin{tabular}{|c|c|c|c|c|c|}
\hline PrEP barriers & $\begin{array}{l}\text { Five survey items related to concerns about } \\
\text { PrEP use, efficacy and stigma; five additional } \\
\text { items assessing potential access barriers } \\
\text { to obtaining PrEP, including lack of health } \\
\text { insurance coverage, difficulty finding a } \\
\text { provider and cost of PrEP. Data are collected } \\
\text { using a Likert scale. }\end{array}$ & $\begin{array}{l}\text { Golub et } a l,{ }^{8} 2012 ; \\
\text { Pulsipher et al, }{ }^{7} 2016\end{array}$ & $x$ & $x$ & $x$ \\
\hline $\begin{array}{l}\text { PrEP efficacy } \\
\text { beliefs }\end{array}$ & $\begin{array}{l}\text { Two-item measure of beliefs regarding the } \\
\text { efficacy of PrEP. Data are collected using a } \\
\text { Likert scale. }\end{array}$ & Pulsipher et al, ${ }^{7} 2016$ & $x$ & $x$ & $x$ \\
\hline $\begin{array}{l}\text { Medication self- } \\
\text { efficacy }\end{array}$ & $\begin{array}{l}\text { Four items assessing self-efficacy to adhere } \\
\text { to PrEP; adapted for PrEP use from the Self- } \\
\text { Efficacy for Appropriate Medication Use Scale. } \\
\text { Data are collected using a Likert scale. }\end{array}$ & $\begin{array}{l}\text { Adapted from Risser et } \\
a^{39}, 2007\end{array}$ & $x$ & $x$ & $x$ \\
\hline $\begin{array}{l}\text { Beliefs about } \\
\text { medicines }\end{array}$ & $\begin{array}{l}\text { Items from the Beliefs about Medicines } \\
\text { Questionnaire (BMQ), including the BMQ- } \\
\text { Specific subscale, a 10-item scale assessing } \\
\text { representations of medications, and the BMC- } \\
\text { General subscale, an eight-item subscale } \\
\text { assessing beliefs about medicines. Data are } \\
\text { collected using a Likert scale. }\end{array}$ & Horne et al ${ }^{40}, 1999$ & $x$ & $x$ & $x$ \\
\hline Sexual behaviours & $\begin{array}{l}\text { Three items measuring over the past } 3 \text { months } \\
\text { the total number of male anal sex partners, } \\
\text { number of partners in which condoms were } \\
\text { not used exclusively and the HIV status of } \\
\text { partners. Data are collected using numeric text } \\
\text { entries. }\end{array}$ & & $x$ & $x$ & $x$ \\
\hline Mental health & $\begin{array}{l}\text { 21-item Depression, Anxiety, Stress Scale } \\
\text { measuring depression, anxiety and stress } \\
\text { over the past week. Data are collected using a } \\
\text { Likert scale. }\end{array}$ & $\begin{array}{l}\text { Lovibond and Lovibond, }{ }^{41} \\
1995\end{array}$ & $x$ & $x$ & $x$ \\
\hline
\end{tabular}


Table 1 Continued

\begin{tabular}{|c|c|c|c|c|c|c|}
\hline Construct & Description of measure & Source & BL & $3 m$ & $6 m$ & 6m EHR \\
\hline Social support & $\begin{array}{l}\text { 8-item PROMIS Informational and } 7 \text {-item } \\
\text { Emotional Support Scales, respectively, } \\
\text { measuring perceived sources of support over } \\
\text { the past month. Data are collected using a } \\
\text { Likert scale. }\end{array}$ & Hahn et al ${ }^{42}, 2014$ & $x$ & $x$ & $x$ & \\
\hline $\begin{array}{l}\text { Patient } \\
\text { satisfaction with } \\
\text { PrEP-related } \\
\text { services }\end{array}$ & $\begin{array}{l}\text { 8-item Client Satisfaction Questionnaire } \\
\text { (CSQ-8) measuring of satisfaction with PrEP- } \\
\text { related healthcare services received. Data are } \\
\text { collected using a Likert scale. }\end{array}$ & $\begin{array}{l}\text { Attkisson and Greenfield, }{ }^{25} \\
2004\end{array}$ & & $x$ & $x$ & \\
\hline $\begin{array}{l}\text { Satisfaction with } \\
\text { interpersonal } \\
\text { relationship with } \\
\text { navigator }\end{array}$ & $\begin{array}{l}\text { For participants randomised to PN intervention } \\
\text { only; nine-item measure of satisfaction with } \\
\text { care provided by a patient navigator. Data are } \\
\text { collected using a Likert scale. }\end{array}$ & $\begin{array}{l}\text { Jean-Pierre et al, } \\
2012\end{array}$ & & $x$ & $x$ & \\
\hline
\end{tabular}

$\begin{array}{llll}\text { PN intervention } & \begin{array}{l}\text { For participants randomised to PN, brief } \\ \text { telephone key informant interview assessing } \\ \text { acceptability of the PN intervention. Data are } \\ \text { qualitative. }\end{array} & \text { Team developed } \\ \text { PrEP initiation } & \begin{array}{l}\text { Up to } 10 \text { survey items assessing whether } \\ \text { patient had received a prescription for PrEP, } \\ \text { decided to fill a PrEP prescription and taken } \\ \text { at least one dose of PrEP since the start of the } \\ \text { study. Categorical data are collected. }\end{array} & \times \\ & \end{array}$

\begin{tabular}{ll}
$\begin{array}{ll}\text { Demographic } \\
\text { characteristics }\end{array}$ & $\begin{array}{l}\text { Demographic items (e.g., age, race, gender, } \\
\text { sexual orientation, relationship status, country } \\
\text { of origin, US citizen status, primary language, } \\
\text { education level, employment status, income } \\
\text { and health insurance status) have categorical } \\
\text { response options. Age data are collected with } \\
\text { numeric text entry. }\end{array}$ \\
Medical & Comorbid medical conditions, medications \\
characteristics & prescribed and history of STI testing. \\
PrEP-related & Date(s) appointment scheduled for PrEP \\
healthcare & consultation; date attended appointment for \\
received & PrEP consultation; date(s) healthcare provider \\
& wrote PrEP prescription(s); date the first \\
& follow-up PrEP appointment(s) scheduled; \\
date(s) of follow-up medical appointment & attended; data recorded in EHR regarding \\
& adherence and discontinuation; other data \\
& regarding PrEP-related healthcare. \\
\hline
\end{tabular}

BL, baseline; EHR, electronic health record; 3m, 3-month follow-up appointment; 6m, 6 month follow-up appointment; PN, patient navigation; PrEP, pre-exposure prophylaxis; STI, sexually transmitted infection.

\section{Medication Event Monitoring System (MEMS) device data collection}

At baseline and 3-month follow-up, all participants will be asked if they have obtained a prescription for PrEP. Those who indicate that they have been prescribed PrEP and have taken at least one pill will be asked to track their PrEP adherence via a MEMS device. ${ }^{30}$ A MEMS device is a standard prescription bottle that includes a larger lid containing a MEMS monitoring cap. During the baseline or at a 3-month follow-up visit, the research coordinator will give participants a MEMS device with instructions for its use. Participants will be instructed to place all PrEP pills into a MEMS container so when a pill is removed, the device will record date and time of removal of the medication dose using a small microcircuit. A medication 'event' is removal of bottle closure, followed by replacement of closure. A study staff member will collect MEMS devices from participants in-person at the 3-month and/ or 6-month follow-up and connect them to a MEMS Reader, which transfers data to a secured server via a secured web platform. Timing adherence will be calculated as percentage of time each participant took PrEP as prescribed by determining number of doses of PrEP taken at 24-hour intervals for a once daily PrEP regimen 
and dividing it by number of days adherence was monitored with MEMS.

\section{Medical record data collection}

We will collect the following data from EHRs 6 months following study enrollment after obtaining a written release of information: (1) date(s) initial appointment to speak to a healthcare provider regarding PrEP was scheduled; (2) date participant attended an initial appointment to discuss PrEP; (3) dates and number of prescriptions written for PrEP by a healthcare provider; (4) date the first follow-up visit related to PrEP was scheduled; and (5) dates the participant attended follow-up appointments related to PrEP. We will document EHR data regarding PrEP initiation and adherence, barriers to PrEP adherence, exams and laboratory tests required to obtain an initial or follow-up PrEP prescription, side effects attributed to PrEP, diagnosis and treatment of illness during the study, prescription medications, comorbid health conditions and history of STI testing. We will also note whether the EHR indicates that participants decided to cease taking PrEP and the date of discontinuation.

\section{PN intervention encounter data}

Using an approach developed by the Patient Navigation Research Program, ${ }^{31}$ patient navigators will record process data related to the PN services provided, including: (1) number of encounters; (2) length of time of each encounter; (3) barriers experienced by patients; and (4) actions taken to reduce barriers. The patient navigators will also be asked to keep detailed qualitative notes regarding telephone, text and in-person encounters with participants, including participants' progress in the PrEP continuum, successes in implementing the PN intervention and challenges in implementing the PN intervention.

\section{Feasibility evaluation}

A feasibility evaluation will examine areas critical to success of a larger research study. First, we will evaluate recruitment strategies by assessing the number of individuals eligible for the study, number with whom we could establish contact, number who agreed to participate or did not, reasons for non-participation, and ineligibility and difficulty with implementing the randomisation process. Second, we will assess methods of data collection including participant time burden for completing baseline surveys, participant difficulty in answering survey questions, difficulty of using MEMS, reliability of surveys, number and types of missing survey data, timing of follow-up data collection and rates of completion of follow-up surveys. Third, we will examine feasibility and processes of delivering the PN intervention by assessing the actions taken by the patient navigators and frequency and type of encounters. Data will be examined descriptively to identify areas for improvement of the study or PN intervention methods before a larger research study is initiated.

\section{Acceptability evaluation}

An extensive acceptability evaluation will examine how both individuals randomised to the PN intervention and those involved in implementing the programme reacted to the intervention. ${ }^{32}$ Data will be collected for the acceptability evaluation using multiple quantitative and qualitative methods, including patient navigator encounter logs, patient navigator client notes, patient navigator supervisor notes, participant surveys, and participant key informant interviews. Quantitative acceptability data will be collected from pilot test participants at the 6-month follow-up using validated surveys assessing satisfaction with PrEP-related healthcare ${ }^{25}$ and Interpersonal Relationship with the Navigator, ${ }^{26}$ as well as from the patient navigators through the PN intervention encounter logs. Qualitative data will be collected from pilot test participants at the 6-month follow-up via three open-ended survey questions assessing the aspect of the PN intervention participants liked the best, the aspect of the PN intervention participants liked the least and one thing that could be changed about the PN intervention ${ }^{25}$ as well as via a brief telephone key informant interview conducted by a trained bilingual research coordinator. The semistructured key informant interviews will be conducted in the participant's preferred language and will use a 28-item standardised interview guide designed using Sekhon $e t a l \mathrm{~s}^{33}$ theoretical framework of acceptability. Interviews will last approximately 45 min and will be recorded, transcribed verbatim and translated (if necessary) into English. In addition, both the patient navigators and the supervisor of the patient navigators will record observations regarding the implementation of the PN intervention, including success and challenges.

\section{Monitoring}

Consistent with NIMH requirements, a data monitoring committee is not required given this is not a phase III clinical trial and because of the low-risk nature of this exploratory study.

\section{DATA ANALYSIS \\ Qualitative data}

Qualitative data (key informant interviews, navigator qualitative notes and supervisor notes) will be saved in a text file to import into NVivo ${ }^{34}$ for analysis. The study principal investigators (PIs) will review the qualitative data using a content analysis technique, which identifies emergent themes occurring during discussion. Code mapping ${ }^{35}$ will be used to develop a preliminary set of codes (themes) corresponding to each potential strength of the PN intervention as well as every potential aspect for improvement. Then, the co-PIs will independently code the remaining data and meet to discuss and achieve consensus in coding. Once coded, the data will be summarised by theme. These data will be used to modify the PN intervention, if necessary, or will provide further 
indication that the PN intervention should be tested in a larger scale study.

\section{Quantitative data}

All quantitative data will be summarised descriptively to determine aspects of the PN intervention that worked well and potential areas of improvement of the PN intervention. Our biostatistician will be blinded to intervention assignment for participants and will conduct statistical analyses using SAS V.9.4. ${ }^{36}$ Univariate statistics will be conducted for outcomes measured at baseline and 6-month follow-up to describe sample characteristics between the two groups (UC vs $\mathrm{PN}$ ). Attrition in the two groups will be compared. Characteristics of individuals with missing values and individuals with no missing values will be studied. Multiple imputation technique will be implemented and sensitivity analysis will be conducted to assess if missing data is at random. Assumptions of statistical tests will be evaluated. For assessing preliminary impact, the primary outcomes are seven behaviours associated with the PrEP care continuum (i.e., appointment scheduled for PrEP consultation; appointment attended for PrEP consultation; PrEP prescription received; PrEP prescription filled; PrEP initiated; self-reported PrEP adherence over the past 7 days; and PrEP follow-up medical appointment attended).

To test the preliminary impact of the intervention, a series of logistic regression models will be conducted, with each of the six binary PrEP continuum primary outcome variables set as a criterion: (1) appointment scheduled for PrEP consultation; (2) appointment attended for PrEP consultation; (3) PrEP prescription received; (4) PrEP prescription filled; (5) PrEP initiated; and (6) PrEP follow-up medical appointment attended. In addition, we hypothesise that receipt of the PN intervention will be associated with increased self-reported PrEP adherence over the past 7 days (also a primary outcome variable). To test the preliminary impact on the two measures of self-reported PrEP adherence, we will conduct two linear regression analyses with intervention group assignment entered as the independent variable and each measure of PrEP adherence entered as the dependent variable. Potential demographic variables (e.g., age) will be assessed for inclusion in the models as well. Finally, linear mixed models will be conducted to examine changes in: (1) barriers to PrEP-related care; (2) PrEP knowledge, (3) PrEP efficacy beliefs; (4) medication beliefs; (5) PrEP attitudes; and (6) medication self-efficacy. Linear mixed models allow us to account for the dependence of the repeated measures. Different covariance structures will be implemented, and the information criterion such as AIC or BIC will be used for model selection. We hypothesise that participants randomised to $\mathrm{PN}$ will have greater engagement in PrEP continuum behaviours compared with participants randomised to UC. Since data analyses are exploratory, and there is not adequate statistical power to evaluate efficacy of the PN intervention, these tests can only provide information regarding data trends and effect sizes. If the study and PN intervention methods are found to be feasible and acceptable, a larger study with adequate statistical power will be conducted to appropriately evaluate the efficacy of the PN intervention.

\section{DISCUSSION}

Young adult Latino MSM are disproportionately at risk for HIV infection. ${ }^{5}$ PrEP has the potential to yield substantial benefits in reducing HIV incidence among vulnerable populations, yet Latino MSM are one of the least likely at-risk groups to use PrEP. ${ }^{737}$ The study described in this paper has the potential to begin to address this ethnic health disparity by pilot testing a peer PN intervention specifically adapted for young adult Latino MSM. Study findings will indicate if, and what types of, revisions should be made to the PN intervention. Potential study limitations include the lack of generalisability to other populations at risk for HIV, the lack of data collection from other community stakeholders, the need for potential participants to be proactive to participate in the study, the lack of concealment of randomisation block size and the potential for loss to follow-up. Results from this pilot trial will inform the design of a subsequent largescale RCT that will be designed to fully test the efficacy of the PN intervention in impacting key gaps in the PrEP continuum, including adherence to PrEP after initiation and long-term retention in PrEP-related care. If subsequently found to be efficacious, this culturally tailored PN PrEP intervention has the potential to be scaled-up throughout federally qualified health centres across the country, which may decrease ethnic disparities seen in HIV.

\section{ETHICS AND DISSEMINATION Ethical consideration}

This study was approved by the San Diego State University institutional review board (Protocol number: HS-2017-0187) and is registered at ClinicalTrials.gov (NCT04048382; https://clinicaltrials.gov/ct2/show/ NCT04048382). All staff are trained on research ethics. Peer navigators are trained on peer navigation and receive confidential weekly supervision by both principal investigators who have experience in clinical supervision and peer navigation training. Confidentiality is ensured at all times during this research process. Identifiable data are only shared with authorised study staff and kept in locked files and password-protected computers; identifiable information will not be shared during dissemination activities. All participants are provided a unique identifier to further protect participants' identity, and all qualitative data are deidentified. Informed consent is obtained from all eligible participants by trained study staff. Participants are able to stop participation in the study at any time. Participants are provided resources to access PrEP and sexual healthcare regardless of the condition to which they are assigned (PN or usual care). All protocol 
modifications will be approved by the San Diego State University institutional review board and described in dissemination efforts on study completion.

\section{Dissemination plan}

The intervention development process and plan, as well as the results of the study, will be shared through peerreviewed journal publication, conference presentations, and healthcare system and community presentations.

\section{Author affiliations}

'Department of Psychology, San Diego State University, San Diego, California, USA ${ }^{2}$ SDSU/UC San Diego Joint Doctoral Program in Clinical Psychology, San Diego, California, USA

${ }^{3}$ Family Health Centers of San Diego, San Diego, California, USA

${ }^{4}$ San Diego State University Research Foundation, San Diego, California, USA

Acknowledgements The authors would like to thank Guillermo Martin, Sergio Velasquez and Sheldon Morris for their support and assistance.

Contributors The authors confirm that they have met the following authorship criteria: (1) substantial contributions to the conception or design of the work (AJB, CBR, C-DL and KJW), or the acquisition, analysis or interpretation of data (AJB, JRG, CBR, C-DL, CMC, KAN, KML, NCL, IJJ, DR, RACA, JPB, MF and KJW); (2) drafted the work or revised it critically for important intellectual content (all authors); (3) gave final approval of the version published (all authors); and (4) agreed to be accountable for all aspects of the work in ensuring that questions related to the accuracy or integrity of any part of the work are appropriately investigated and resolved (all authors).

Funding Research reported in this publication was supported by the National Institute of Mental Health of the National Institutes of Health under Award Number R34MH114699. NL's efforts on this research were supported by the National Cancer Institute of the National Institutes of Health under award numbers U54CA12384 and U54CA132379. IJJ's and DR's efforts on this research were supported by the National Cancer Institute of the National Institutes of Health under award number R25CA132699 and under the National Institutes of Health Initiative for Maximising Student Development, under award number 5R25GM058906.

Disclaimer The content is solely the responsibility of the authors and does not represent the official views of the National Institutes of Health.

Competing interests None declared.

Patient consent for publication Not required.

Provenance and peer review Not commissioned; externally peer reviewed.

Supplemental material This content has been supplied by the author(s). It has not been vetted by BMJ Publishing Group Limited (BMJ) and may not have been peer-reviewed. Any opinions or recommendations discussed are solely those of the author(s) and are not endorsed by BMJ. BMJ disclaims all liability and responsibility arising from any reliance placed on the content. Where the content includes any translated material, BMJ does not warrant the accuracy and reliability of the translations (including but not limited to local regulations, clinical guidelines, terminology, drug names and drug dosages), and is not responsible for any error and/or omissions arising from translation and adaptation or otherwise.

Open access This is an open access article distributed in accordance with the Creative Commons Attribution Non Commercial (CC BY-NC 4.0) license, which permits others to distribute, remix, adapt, build upon this work non-commercially, and license their derivative works on different terms, provided the original work is properly cited, appropriate credit is given, any changes made indicated, and the use is non-commercial. See: http://creativecommons.org/licenses/by-nc/4.0/.

\section{ORCID iDs}

Sarah A Rojas http://orcid.org/0000-0003-0268-1682

Nicholas C Lucido http://orcid.org/0000-0002-7426-213X

\section{REFERENCES}

1 Healthy People. Topics and objectives: HIV 2016, 2020. Available: https://www.healthypeople.gov/2020/topics-objectives/topic/hiv/ objectives [Accessed 05 Jan 2017].
2 AIDS.gov. National HIV/AIDS strategy: overview. Available: www. aids.gov/federal-resources/national-hiv-aids-strategy/overview/ [Accessed 05 Jan 2017].

3 Centers for Disease Control and Prevention. HIV and Gay and Bisexual Men, 2018. Available: www.cdc.gov/hiv/pdf/group/msm/ cdc-hiv-msm.pdf [Accessed 02 Feb 2019].

4 Smith DK, Van Handel M, Wolitski RJ, et al. Vital Signs: Estimated Percentages and Numbers of Adults with Indications for Preexposure Prophylaxis to Prevent HIV Acquisition--United States, 2015. MMWR Morb Mortal Wkly Rep 2015;64:1291-5.

5 Centers for Disease Control and Prevention. Diagnoses of HIV infection in the United States and dependent areas, 2014. In: HIV surveillance report. , 2015: 26, 1-123.

6 Anderson PL, Glidden DV, Liu A, et al. Emtricitabine-tenofovir concentrations and pre-exposure prophylaxis efficacy in men who have sex with men. Sci Transl Med 2012;4:151ra25-51ra25.

7 Pulsipher CA MJ, Plant A, Curtis P. Addressing PreP dispartities among young gay and bisexual men in California: California HIVIAIDS research program. 41, 2016.

8 Golub SA, Gamarel KE, Rendina HJ, et al. From efficacy to effectiveness: facilitators and barriers to PreP acceptability and motivations for adherence among MSM and transgender women in New York City. AIDS Patient Care STDS 2013;27:248-54.

9 Bauermeister JA, Meanley S, Pingel E, et al. Prep awareness and perceived barriers among single young men who have sex with men. Curr HIV Res 2013;11:520-7.

10 Ayala G, Makofane K, Santos G-M, et al. Access to basic HIV-related services and PreP acceptability among men who have sex with men worldwide: barriers, facilitators, and implications for combination prevention. J Sex Transm Dis 2013;2013:1-11.

11 Kimball D, Rivera D, Gonzales M, et al. Medical Mistrust and the PreP cascade among Latino sexual minority men. AIDS Behav 2020;24:3456-61.

12 Rivera DB, Brady JP, Blashill AJ. Traditional Machismo, Caballerismo, and the pre-exposure prophylaxis (PreP) cascade among a sample of Latino sexual minority men. J Sex Res 2021;58:21-8.

137 th International conference on HIV treatment and prevention adherence. The spectrum of engagement in HIV prevention: proposal for a PreP cascade. Miami Beach, Florida: 7th International conference on HIV treatment and prevention adherence, 2012.

14 Braschi CD, Sly JR, Singh S, et al. Increasing colonoscopy screening for Latino Americans through a patient navigation model: a randomized clinical trial. J Immigr Minor Health 2014;16:934-40.

15 Enard KR, Nevarez L, Hernandez M, et al. Patient navigation to increase colorectal cancer screening among Latino Medicare enrollees: a randomized controlled trial. Cancer Causes Control 2015;26:1351-9.

16 Wilson FA, Villarreal R, Stimpson JP, et al. Cost-Effectiveness analysis of a colonoscopy screening navigator program designed for Hispanic men. J Cancer Educ 2015;30:260-7.

17 Family Health Centers of San Diego. Welcome to gay men's health service, 2015. Available: https://gaymenshealth.org/ [Accessed 11 Feb 2019].

18 Whitehead AL, Julious SA, Cooper CL, et al. Estimating the sample size for a pilot randomised trial to minimise the overall trial sample size for the external pilot and main trial for a continuous outcome variable. Stat Methods Med Res 2016;25:1057-73.

19 United States Census Bureau. QuickFacts: San Diego County, California, 2019. Available: https://www.census.gov/quickfacts/fact/ table/sandiegocountycalifornia,ca/PST045218 [Accessed 13 Apr 2019].

20 Newport F, Gates G. San Francisco Metro area ranks highest in LGBT percentage, 2015. Available: http://www.gallup.com/poll/ 182051/san-francisco-metro-area-ranks-highest-lgbt-percentage. aspx [Accessed 01 Jun 2016].

21 US Public Health Service. Preexposure prophylaxis for the prevention of HIV infection in the United States-2014: a clinical practice guideline, 2014. Available: www.cdc.gov/hiv/pdf/prepguidelines2014. pdf [Accessed 05 Jan 2017].

22 Medsharing, Inc. Randomizer for Clinical Trial [program. Fontenay Sous Bois, France: Medsharing, Inc, 2016.

23 Bartholomew LK, Parcel GS, Kok G. Planning health promotion programs: an intervention mapping approach. Third ed. San Francisco, California: Jossey-Bass, 2011.

24 Calhoun EA, Whitley EM, Esparza A, et al. A national patient navigator training program. Health Promot Pract 2010;11:205-15.

25 Attkisson CC, Greenfield TK. The UCSF Client Satisfaction Scales: I. The Client Satisfaction Questionnaire-8. In: Maruish ME, ed. The use of psychological testing for treatment planning and outcomes assessment. 3rd ed. Mahwah, New Jersey: Lawrence Erlbaum Associates, 2004. 
26 Jean-Pierre P, Fiscella K, Winters PC, et al. Psychometric development and reliability analysis of a patient satisfaction with interpersonal relationship with navigator measure: a multi-site patient navigation research program study. Psychooncology 2012;21:986-92.

27 McKenna SP, Doward LC. The translation and cultural adaptation of patient-reported outcome measures. Value Health 2005;8:89-91.

28 Swaine-Verdier A, Doward LC, Hagell P, et al. Adapting quality of life instruments. Value Health 2004;7 Suppl 1:S27-30.

29 Epstein J, Santo RM, Guillemin F. A review of guidelines for cross-cultural adaptation of questionnaires could not bring out a consensus. J Clin Epidemiol 2015;68:435-41.

30 AARDEX. MEMS, 2010. Available: http://www.aardexgroup.com/ aardex_index.php?group=aardex [Accessed 15 Sep 2010].

31 Katz ML, Young GS, Reiter PL, et al. Barriers reported among patients with breast and cervical abnormalities in the patient navigation research program: impact on timely care. Womens Health Issues 2014;24:e155-62.

32 Bowen DJ, Kreuter M, Spring B, et al. How we design feasibility studies. Am J Prev Med 2009;36:452-7.

33 Sekhon M, Cartwright M, Francis JJ. Acceptability of health care interventions: a theoretical framework and proposed research agenda. Wiley Online Library, 2018.

34 QSR International. NViVo 10 For Windows [program, 2014.
35 Knodel J. The design and analysis of focus group studies: a practical approach. successful focus groups: advancing the state of the art. London: Sage Publications, 1993: 35-50.

36 SAS Institute. SAS software version 9.4 [program. Cary, NC, 2013.

37 Grant RM, Lama JR, Anderson PL, et al. Preexposure chemoprophylaxis for HIV prevention in men who have sex with men. N Engl J Med 2010;363:2587-99.

38 Mustanski B, Ryan DT, Hayford C, et al. Geographic and individual associations with PreP stigma: results from the radar cohort of diverse young men who have sex with men and transgender women. AIDS Behav 2018;22:3044-56.

3938 Risser J, Jacobson TA, Kripalani S. Development and psychometric evaluation of the self-efficacy for appropriate medication use scale (SEAMS) in low-literacy patients with chronic disease. J Nurs Meas 2007;15:203-19.

40 Horne R, Weinman J, Hankins M. The beliefs about medicines questionnaire: the development and evaluation of a new method for assessing the cognitive representation of medication. Psychol Health 1999;14:1-24.

41 Lovibond SH, Lovibond PF. Manual for the depression anxiety stress scales. 2nd. Sydney: Psychology Foundation of Australia, 1995.

42 Hahn EA, DeWalt DA, Bode RK, et al. New English and Spanish social health measures will facilitate evaluating health determinants. Health Psychol 2014;33:490-9. 